\title{
Indices cefálico total y corporal de la vicuña (Vicugna vicugna mensalis) adulta en el Centro de Investigación, Producción y Transferencia Tecnológica Tullpacancha - Huancavelica
}

\section{Cephalic total and corporal indexes of the mature vicuña (Vicugna vicugna mensalis) in the Center of Investigation, Production and Technological Transfer Tullpacancha - Huancavelica}

\author{
Mercedes J. Guillén Quispe, Lyana Quispe Ochoa, ${ }^{* *}$ Maite Baquerizo Revilla ${ }^{* * *}$ \\ http://dx.doi.org/10.21503/CienciayDesarrollo.2011.v14.05
}

\section{RESUMEN}

El objetivo del estudio fue determinar el índice cefálico total y corporal en la vicuña (Vicugna vicugna mensalis) adulta. La investigación se llevó a cabo en el Centro de Investigación, Producción y Transferencia Tecnológica (CIPTT) Tullpacancha, en la provincia de Churcampa, departamento de Huancavelica, a una altitud de $4150 \mathrm{msnm}$, durante la actividad del chaccu, en julio de 2009. Se seleccionaron 100 vicuñas adultas ( 50 hembras y 50 machos), a las que se tomaron las medidas de ancho y largo de la cabeza para determinar el índice cefálico total, así como también el largo del tronco y el perímetro torácico para determinar el índice corporal, haciendo uso de instrumentos zoométricos. El ancho de la cabeza se tomó con la escuadra de dos ramas, y el largo de la cabeza, el largo del tronco y el perímetro torácico, con cinta métrica. Los resultados mostraron que el índice cefálico total para hembras y machos fue de $56,81 \% \pm$ 1,64 y $56,85 \% \pm 2,35$ respectivamente, en tanto que el índice corporal en hembras fue de $89,91 \% \pm 1,22$, y en machos, $90,22 \% \pm 1,42$. Se concluye que hembras y machos se clasifican dentro de los braquicéfalos, según el índice cefálico total, y como sublonguilíneos, según su índice corporal. No se encontraron diferencias notables entre hembras y machos.

Palabras clave: biometría, cráneo, cuerpo, camélido sudamericano.

\section{ABSTRACT}

The purpose of this study was to determine the total cephalic index and the corporal index in adult vicuña (Vicugna vicugna mensalis) in the Center of Investigation, Production and Technological Transfer Tullpacancha - Huancavelica with an altitude of 4150 meters above sea level, during the chaccu in July 2009. The investigation took from July 2009 to February 2011. 100 adult vicuñas (50 females and 50 males) were selected. The measures of width and length of the head were taken to determine the total cephalic index; the same was done with the body length and thoracic perimeter in order to determine the corporal index, using zoometrical instruments. The width of the head was taken with the pliers and the length of the head, the body length and the thoracic perimeter with the metric tape. The data was written recorded and processed using the mathematical formula to obtain the total cephalic index and the corporal index. The results show that the total cephalic index for females and males was $56,81 \% \pm 1,64$ y $56,85 \%$ $\pm 2,35$ respectively, the corporal index in females was $89,91 \% \pm 1,22$ and for males was $90,22 \% \pm 1,42$. In conclusion, females and males classify as brachycephalous according to the total cephalic index and as sub-dolichomorpho according to the corporal index. There are no differences among females and males.

Key words: biometria, cranium, body, southamerican camelid.

* Médica veterinaria graduada en la EAP de Medicina Veterinaria de la UAP.

** Docente de la EAP de Medicina Veterinaria de la UAP.

*** Docente de la EAP de Medicina Veterinaria de la UAP. (maitebaquerizo@yahoo.es) 


\section{INTRODUCCIÓN}

La vicuña constituye un recurso de importancia social, económica, cultural y científica para el Perú y algunos de los países de la Región Andina(1). Entre los 3800 y 4800 msnm aprovecha eficientemente los pastos nativos, lo que determina bajos costos de producción por ser una especie rústica y adaptada en forma completa a su hábitat y por no competir con el ganado doméstico, cuya crianza es limitada en estas áreas(2). Wheeler reconoce dos subespecies geográficas: una al sur, la Vicugna vicugna vicugna, y otra al norte, la Vicugna vicugna mensalis. La especie del sur es la de mayor tamaño y de color más claro con respecto a la especie del norte. Sin embargo, la más estudiada es la Vicugna vicugna mensalis(3).

En la comunidad de Tullpacancha (Huancavelica) se realiza la crianza de vicuñas (Vicugna vicugna mensalis) en semicautiverio, siguiendo un método compatible con la introducción de técnicas de mejoramiento, para lo cual ha sido necesario determinar las diferentes medidas zoométricas y su relación a través de índices para la caracterización de la especie. Se debe tener en consideración que el índice cefálico tiene una importancia etnológica, sobre todo porque su variación no está influenciada por los factores ambientales ni por el manejo que reciben los animales(4). El índice corporal provee de cifras claras que determinan el significado de braquimorfo, mesomorfo y dolicomorfo(5). Los valores referenciales zoométricos destacan que a un menor valor de índice corporal, la morfología del animal se aproxima a un rectángulo, forma que predomina en los animales de aptitud carnicera(6).

El estudio tiene por objetivo determinar el índice cefálico total y corporal en la vicuña (Vicugna vicugna mensalis) adulta en el Centro de Investigación, Producción y Transferencia Tecnológica (CIPTT) Tullpacancha, en la provincia de Churcampa, departamento de Huancavelica, como punto de partida para estudios posteriores con la finalidad de establecer un estándar zoométrico tendiente a su mejor aprovechamiento.

\section{MATERIALES Y MÉTODOS}

Este estudio se realizó en el Centro de Investigación, Producción y Transferencia Tecnológica (CIPTT) Tullpacancha, en la provincia de Churcampa, departamento de Huancavelica. La toma de muestra se realizó durante el chaccu, en el mes de julio del 2009, y el análisis de la misma se realizó en la Escuela Académico Profesional de Medicina Veterinaria de la Universidad Alas Peruanas (UAP), en Pachacámac, Lima.

La población de vicuñas del CIPTT - Tullpacancha es de 1059 cabezas, y se tomaron medidas de 100 vicuñas adultas (50 machos y 50 hembras). Se realizaron las mediciones de largo y ancho de la cabeza, perímetro torácico y largo del tronco, para obtener el índice cefálico total y el índice corporal respectivamente. Los instrumentos que se utilizaron fueron la escuadra de dos ramas y la cinta métrica.

El índice cefálico total es la relación que existe entre el ancho de la cabeza (medida desde la parte externa de un arco zigomático al otro extremo) multiplicada por cien y el largo de la cabeza (medida tomada desde el apófisis occipital externo al margen súpero anterior de la nariz)(5). Los tipos de cabeza se clasifican en tres clases: dolicocéfalo (propio del animal de cabeza alargada, y cuya resultante del índice cefálico total es menor de $46 \%$ ), mesocéfalo (característico del animal de cabeza intermedia, y cuya resultante del índice cefálico total está entre 46 y 55\%), y braquicéfalo (que define al animal de cabeza corta, y cuya resultante del índice cefálico total es mayor a $55 \%)(5)$. 
Tabla 1. Clasificación de tipos y subtipos morfológicos según Luquet, de acuerdo al índice corporal (IC)

\begin{tabular}{l|l|c}
\multirow{2}{*}{$\begin{array}{c}\text { TIPOS } \\
\text { MORFOLÓGICOS }\end{array}$} & \multicolumn{1}{c}{$\begin{array}{c}\text { SUBTIPOS } \\
\text { MORFOLÓGICOS }\end{array}$} & $\begin{array}{c}\text { ÍNDICE CORPORAL } \\
\text { (IC) \% }\end{array}$ \\
\cline { 2 - 2 } Braquimorfos & Ultrabrevilíneos & 60 \\
& Brevilíneos & 65 \\
& Subbrevílineos & 70 \\
\hline Mesomorfo & Mediolíneos & 71 a 84 \\
\hline \multirow{2}{*}{ Dolicomorfo } & Sublonguilíneo & 85 \\
& Longuilíneos & 92,5 \\
& Ultralonguilíneos & 100 \\
\hline
\end{tabular}

Fuente: Maniero.(5)

El índice corporal se obtiene tomando el largo total del tronco, medido desde la punta de la articulación escápulo- humeral hasta la tuberosidad isquiática, multiplicado por cien y dividido por el perímetro torácico, tomado desde la línea a la cruz pasando en forma tangencial por detrás de los codos(5). Del índice corporal, según indica Maniero, fue Giuliani quien creó la subdivisión de los parámetros, que fue rectificada más tarde por Luquet(5).

\section{RESULTADOS}

De las mediciones de los 50 machos y 50 hembras, se obtuvieron los resultados en promedio y desviación estándar. Así, el largo medio de cabeza para el caso de hembras y machos fue de 25,00 $\mathrm{cm} \pm 1,31$ y $25,70 \mathrm{~cm} \pm 1,28$ respectivamente; para el ancho de cabeza, se reportaron valores medios de $14,19 \mathrm{~cm} \pm 0,58$ para hembras y 14,59 $\mathrm{cm} \pm 0,58$ para machos; el largo de tronco para hembras fue de $80,88 \mathrm{~cm} \pm 1,52$, y para machos, de $81,62 \mathrm{~cm} \pm 1,48$; por su parte, el perímetro torácico en hembras resultó de $89,96 \mathrm{~cm} \pm 1,31$, y en machos, $90,48 \mathrm{~cm} \pm 1,58$.

El índice cefálico total y el índice corporal se observan en la tabla 2.

Los valores de los índices cefálico total y corporal en vicuñas hembras y machos adultas del CIPTT Tullpacancha se clasifican como braquicéfalos y sublonguilíneos.

Tabla 2. Medidas biométricas (cm) e índices cefálico total (\%) y corporal (\%) de vicuñas adultas según sexo, en Tullpacancha - Huancavelica 2011

\begin{tabular}{r|c|c|c|c|c|c}
\multicolumn{1}{c|}{ SEXO } & $\begin{array}{c}\text { LARGO DE } \\
\text { CABEZA }\end{array}$ & $\begin{array}{c}\text { ANCHO DE } \\
\text { CABEZA }\end{array}$ & $\begin{array}{c}\text { ÍNDICE } \\
\text { CEFÁLICO }\end{array}$ & $\begin{array}{c}\text { LARGO DE } \\
\text { CUERPO }\end{array}$ & $\begin{array}{c}\text { PERÍMETRO } \\
\text { TORÁCICO }\end{array}$ & $\begin{array}{c}\text { ÍNDICE } \\
\text { CORPORAL }\end{array}$ \\
\hline Hembras & 25,00 & 14,19 & $56,81 \pm 1,64$ & 80,88 & 89,96 & $89,91 \pm 1,22$ \\
\hline Machos & 25,70 & 14,59 & $89,91 \pm 1,22$ & 81,62 & 90,48 & $90,22 \pm 1,42$ \\
\hline
\end{tabular}




\section{DISCUSIÓN}

En la investigación, las hembras presentaron un índice cefálico total medio de 56,81\% \pm 1,64, y los machos, $56,85 \% \pm 2,35$; asimismo, se encontró un índice corporal medio en hembras de $89,91 \% \pm 1,22$, y en machos, uno de $90,22 \% \pm$ 1,42. Los resultados encontrados en las vicuñas de Tullpacancha discrepan con aquellos obtenidos en el trabajo de investigación en vicuñas adultas de la hacienda Cala Cala (tabla 3), que muestra valores del índice cefálico total en hembras de 51,76 \pm 0,45 , y en machos, de $52,98 \pm 0,39$, en tanto que con respecto al índice corporal muestra valores en hembras de $85,46 \pm 0,74$, y en machos, 83,20 $\pm 0,59(7)$. Si bien no se encuentran coincidencias para las variables índice cefálico y corporal, esto se puede deber a los diferentes métodos empleados, los instrumentos de precisión y la diferente ubicación geográfica. Cabe señalar que en el presente trabajo se evaluaron 100 vicuñas con instrumentos de precisión, y que las medidas fueron tomadas por personal capacitado apoyado por asistentes de sujeción, lo que ayudó al manejo de estos animales de naturaleza nerviosa. En el estudio realizado en Puno (7), no se mencionan los instrumentos utilizados y más bien manifiesta dificultad en el manejo. En lo referente a la ubicación geográfica, la presente investigación muestra datos de Huancavelica, mientras que los datos de Dianderas fueron de Puno. Es importante señalar esto porque estudios recientes señalan que entrelasvicuñas peruanasse determinóla existencia de cuatro grupos demográfica y genéticamente distintos. En relación con el indicador largo de la cabeza, la presente investigación muestra como valor medio $25,00 \mathrm{~cm} \pm 1,31$ para hembras y 25,70 $\mathrm{cm} \pm 1,28$ para machos. Estos resultados discrepan de los de Dianderas, que establece una medida de $23,15 \mathrm{~cm}$ para hembras, $\mathrm{y} 24,85 \mathrm{~cm}$ para machos. Sin embargo, hay coincidencias con estudios $(8)$ que consignan valores de $26 \mathrm{~cm}$ para ambos sexos. En lo referente al ancho de la cabeza, la presente investigación ha determinado una media de $14,19 \mathrm{~cm} \pm 0,58$ para hembras y $14,59 \mathrm{~cm} \pm 0,53$ para machos, discrepando con los resultado de Dianderas, que en machos obtiene $13,17 \mathrm{~cm}$ y en hembras $11,49 \mathrm{~cm}$.

En lo referente al largo del tronco, la investigación muestra $80,88 \mathrm{~cm} \pm 1,52$ para hembras y $81,62 \mathrm{~cm} \pm 1,48$ para machos, discrepando significativamente con los datos presentados por Dianderas, que establece valores de 75,27 cm en hembras y 75,22 en machos, y también con los presentados por el Instituto Nacional de Innovación Agraria (INIA), que consigna $62,28 \mathrm{~cm} \pm 5,69$ para hembras y 61,46 $\pm 3,11$ para machos. En lo referente a perímetro torácico, la investigación señala $89,96 \mathrm{~cm} \pm 1,31$ para hembras y $90,48 \mathrm{~cm} \pm 1,58$ para machos, frente a Dianderas, que encontró $87,83 \mathrm{~cm}$ para hembras y $90,51 \mathrm{~cm}$ para machos, en tanto que el INIA menciona $88,94 \mathrm{~cm} \pm 3,64$ para hembras y $90,06 \pm 2,78$ para machos. En relación a las hembras, se encuentran coincidencias con los datos de INIA y discrepancias con los de Dianderas; sin embargo, respecto de los machos existe coincidencia entre Dianderas, el INIA y la presente investigación.

Tabla 3. Índices cefálico total (\%) y corporal (\%) de vicuñas (Vicugna vicugna mensalis) en Tullpacancha y Cala Cala

\begin{tabular}{c|c|c|c|c}
\multirow{2}{*}{ SEXO } & \multicolumn{2}{|c|}{ ÍNDICE CEFÁLICO TOTAL } & \multicolumn{2}{c}{ ÍNDICE CORPORAL } \\
\cline { 2 - 3 } \cline { 5 - 5 } & TULLPACANCHA & CALA CALA & TULLPACANCHA & CALA CALA \\
\hline \multirow{2}{*}{ Hembras } & $56,81 \pm 1,64$ & $51,76 \pm 0,45$ & $89,91 \pm 1,22$ & $85,70 \pm 0,74$ \\
\hline Machos & $56,85 \pm 2,35$ & $52,98 \pm 0,39$ & $90,22 \pm 1,42$ & $83,20 \pm 0,59$ \\
\hline
\end{tabular}




\section{CONCLUSIÓN}

Los índices zoométricos estudiados en vicuñas hembras y machos de la especie Vicugna vicugna mensalis difieren con los de otras investigaciones realizadas sobre biometría. Sin embargo, los valores encontrados nos permiten clasificar a las vicuñas (Vicugna vicugna mensalis) en braquicéfalos, según el índice cefálico total, y en sublonguilíneos, según el índice corporal. No se encontraron diferencias entre hembras y machos.

\section{REFERENCIAS BIBLIOGRÁFICAS}

1. Fernández-Baca S. Situación actual de los camélidos sudamericanos en Perú. FAO, Lima, Perú, 2005.

2. Zúñiga M. La vicuña. Editorial UAP, Lima, Perú, 2007.

3. Wheeler J. Historia natural de la vicuña. En: http://janecwheeler.com/publications/ Historia\%20Natural\%20de\%20la\%20Vicuna.pdf. Acceso el 20 marzo de 2011.
4. Herrera M., Luque M. Morfoestructura y sistemas para el futuro en la valoración morfológica. Editorial Sañudo, Madrid, España, 2009.

5. Maniero E. Zoometría. Introducción a la morfología externa canina. Editorial UAP, Lima, Perú, 2006.

6. Parés C. Valoración morfológica de animales domésticos. Editorial Sañudo, Madrid, España, 2009.

7. Dianderas A. Contribución al estudio bistórico, descriptivo y zoométrico de la vicuña [tesis para obtener el título de médico veterinario]. Universidad Nacional Mayor de San Marcos, Lima, 1954.

8. Hoffmann R., Ohe K., Ponce C., Ríos M. El manejo de las vicuñas silvestres. Editorial GTZ, Eschborn, Alemania, 1983.

9. INIA. Características biométricas y niveles de testosterona en vicuñas. En: http://www. inia.gob.pe/boletin/boletin0013/carbio. htm. Acceso el 1 octubre de 2008. 